\title{
Bioinformatics analysis of small RNAs in Helicobacter pylori and the role of NAT-67 under tinidazole treatment
}

\author{
JIE DU ${ }^{1,2 *}$, WANG ZHANG ${ }^{2,3^{*}}$, XIAO-HUI LI $^{2}$ and YUAN-JIAN LI ${ }^{2}$ \\ ${ }^{1}$ Department of Pharmacy, Xiangya Hospital, Central South University, Changsha, Hunan 410008; \\ ${ }^{2}$ Department of Pharmacology, School of Pharmaceutical Sciences, Central South University, Changsha, Hunan 410078; \\ ${ }^{3}$ Department of Pharmacy, The First People's Hospital of Changde City of Pharmaceutical Sciences, \\ Changde, Hunan 415003, P.R. China
}

Received September 10, 2019; Accepted April 30, 2020

DOI: $10.3892 / \mathrm{mmr} .2020 .11232$

\begin{abstract}
Helicobacter pylori (Hp) infection is a major cause of gastrointestinal disease. However, the pathogenesis of gastric mucosa injury by $\mathrm{Hp}$ has remained elusive. Small non-coding RNA (sRNA) is a type of widespread RNA in prokaryotic organisms and regulates bacterial growth, reproduction and virulence. In the present study, Hp sRNA profiles were generated to reveal the sequences and possible functions of sRNA by bioinformatics analysis. The role of sRNA in tinidazole (TNZ) treatment was also explored. Total sRNAs of HP26695 were sequenced using an Illumina HiSeq2000. Detected Tags were then compared with a known sRNA database to build an sRNA profile. Reverse transcription-quantitative (RT-q) PCR products were sequenced directly and agarose gel electrophoresis was used to identify NAT-67 and 5'ureB-sRNA in HP. Furthermore, HP was treated with TNZ for 6, 12 and $24 \mathrm{~h}$. The bacterial concentration was measured, the expression of NAT-67, 5'ureB-sRNA and ceuE was determined by RT-qPCR and superoxide dismutase (SOD) activity and reactive oxygen species (ROS) production were detected. A total of 163 sRNA tags were predicted in Hp through bioinformatics analysis. Among them, 35 tags were evolutionarily aconserved
\end{abstract}

Correspondence to: Professor Yuan-Jian Li, Department of Pharmacology, School of Pharmaceutical Sciences, Central South University, 110 Xiang-Ya Road, Changsha, Hunan 410078, P.R. China

E-mail: yuan_jianli@sina.com

*Contributed equally

Abbreviations: Hp, Helicobacter pylori; TNZ, tinidazole; sRNA, small non-coding RNA; ROS, reactive oxygen species; SOD, superoxide dismutase; CagA, cytotoxic-associated gene A; E. coli, Escherichia coli; VacA, vacuolated cytotoxic A; S. aureus, Staphylococcus aureus; AM, antisense mRNA; P. aeruginosa, Pseudomonas aeruginosa; IGR, intergenic region

Key words: Helicobacter pylori, small RNA, NAT-67, tinidazole, oxidative stress in different Hp strains. By target prediction, it was indicated that certain candidate sRNAs were associated with bacterial oxidative stress, virulence and chemotaxis. It was also observed that NAT-67 and 5'ureB-sRNA were downregulated in TNZ-treated HP. TNZ treatment inhibited the growth of Hp, which was accompanied by downregulation of ceuE and SOD activity, as well as upregulation of ROS. RNA sequencing and bioinformatics are valuable in predicting the expression profile and function of sRNA in HP. sRNA-targeted genes may be associated with virulence, oxidative stress and chemokines. Downregulation of NAT-67 by TNZ may be involved in Hp oxidative stress regulation, which may comprise one of the mechanisms of the antibacterial effects of TNZ.

\section{Introduction}

Helicobacter pylori (Hp), a gram-negative bacterium, thrives in the acidic environment of the stomach and is the major risk factor for chronic gastric ulcer and gastric cancer. Hp secretes virulence factors, including cytotoxic-associated gene A (CagA), vacuolated cytotoxic A (VacA) and urease, which cause DNA damage and cell vacuolization of gastric mucosa cells, and then destroy tight junctions between cells and damage the cell barrier by inducing oxidative stress and activating the inflammatory response $(1,2)$. In addition to Hp virulence proteins, it has been suggested that small non-coding RNA may regulate mRNA stability and protein translation, which finally affect bacterial growth and reproduction and contribute to virulence.

Bacterial small non-coding RNA (sRNA), 50-500 nt nucleotides in length, is a type of widespread RNA in prokaryotic organisms, similar to microRNA (miRNA/miR) in eukaryotes. Through nucleotide pairing, sRNA is able to target mRNAs and regulate mRNA stability and protein translation, which finally affects bacterial growth and reproduction and contributes to virulence. Since 1967, the first sRNA 6S RNA was isolated from Escherichia coli (E. coli) and hundreds of sRNAs have been identified in $E$. coli and other bacteria $(3,4)$. However, to date, only 7 sRNAs have been identified in $\mathrm{Hp}$, but research is still in its infancy (5-9). For instance, a study has reported that acid-sensing chemotaxis receptor TlpB, which has a vital role in colonization, was 
upregulated in repG (HPnc5490) deletion mutant Hp (6). Hp 5'ureB-sRNA, a cis-encoded antisense small RNA regulated by the HP0165-HP0166 two-component system, enhanced the expression of ure $\mathrm{AB}$, consequently protecting it from an acid environment $(7,10)$. Another prediction suggested that Hp sRNA NAT-67 was completely complementary to HP1561 (ceuE), a periplasmic iron-binding protein that may regulate oxidative stress (8). All of these studies suggested that sRNAs have critical roles in the growth, colonization and invasion of Hp. Bioinformatics prediction suggested that there may be hundreds of sRNAs in Hp (5). However, the full extent of sRNAs in bacterial chromosomes, as well as their types and functions, remain elusive. Investigating the sRNA expression spectrum of HP is crucial to reveal the biological functions of sRNA and further anti-bacterial therapy.

sRNA has been indicated to be involved in the response of bacteria to environmental fluctuations, including oxidative stress and antibiotic drug exposure. The levels of reactive oxygen species (ROS) were significantly elevated in small RNAs RyhB-1 and RyhB-2 deletion mutant Salmonella Typhimurium, proving RyhB to be an anti-oxidative stress factor (11). Analysis of the small RNA transcriptional response in multidrug-resistant Staphylococcus aureus (S. aureus) suggested that 39 sRNAs were significantly changed by four major classes of antibiotics (12). Furthermore, sRNA SprX (alias RsaOR) shaped bacterial resistance to glycopeptides, an invaluable treatment for methicillin-resistant staphylococcal infections (13). Azithromycin inhibits the expression of small RNAs RsmY and RsmZ in Pseudomonas aeruginosa (P. aeruginosa), hence affecting quorum sensing and biofilm formation, which may be a bactericidal mechanism (14). That is to say that sRNA may contribute to the regulation of sensitivity to antibiotic exposure and resistance to oxidative stress. It is well-known that tinidazole (TNZ) is commonly used for HP treatment by inducing oxidative stress, but the exact mechanism has remained to be fully elucidated. It was hypothesized that SRNA from HP is involved in the regulation of oxidative stress induced by TNZ.

In the present study, an HP sRNA profile was generated to predict the possible functions of sRNA by using bioinformatics analysis, aiming to explore the role of sRNA in HP with TNZ exposure.

\section{Materials and methods}

Bacterial strain and growth conditions. The HP stains 26695 and SS1 were obtained from the American Type Culture Collection. Primary plate cultures of HP were grown from glycerol stocks on trypticase soy agar (TSA) plates with 5\% sheep blood (Thermo Fisher Scientific, Inc.) for 2-3 days in a microaerobic environment $\left(5 \% \mathrm{O}_{2}, 10 \% \mathrm{CO}_{2}, 85 \% \mathrm{~N}_{2}\right)$ at $37^{\circ} \mathrm{C}$. The overnight culture of HP26695 on TSA plates supplemented with $5 \%$ sheep blood was suspended in brain heart infusion medium containing $12 \%$ sheep blood to an optical density of $10^{6}$ colony-forming units $(\mathrm{CFU}) / \mathrm{ml}$ with shaking slowly under microaerobic conditions at $37^{\circ} \mathrm{C}$. When necessary, the following antibiotics were added at the indicated final concentrations: Vancomycin $(50 \mu \mathrm{g} / \mathrm{ml})$, polymyxin B $(10 \mathrm{U} / \mathrm{ml})$, amphotericin $\mathrm{B}(125 \mu \mathrm{g} / \mathrm{ml})$ and trimethoprim (25 $\mu \mathrm{g} / \mathrm{ml}$; all from Melone Pharmaceutical Co., Ltd.). HP in the logarithmic phase was treated with TNZ $\left(10^{-5}, 3 \times 10^{-5}\right.$, $10^{-4} \mathrm{M}$ ) and then subjected to microaerobic conditions at $37^{\circ} \mathrm{C}$ for $24 \mathrm{~h}$. The logarithmic phase of bacteria is a growth peak, which is able to avoid the effect of bacterial death on the results.

RNA preparation. Total RNA was isolated from HP strains using TRIzol reagent (Takara Biotechnology Co., Ltd.). The concentration and purity of eluted RNA was determined spectrophotometrically (Beckman Coulter, Inc.) [optical density at $260 \mathrm{~nm}(\mathrm{OD} 260) / \mathrm{OD} 280$ ratio between 1.8 and 2.2]. The ribosomal RNA was removed prior to deep sequencing.

Bioinformatics analysis by The Beijing Genomics Institute. Total RNA from HP26695 was sequenced using an Illumina HiSeq2000 (Ilumina, Inc.). The reads were spliced based on overlap relations. According to their genomic locations, tags in the intergenic region (IGR) and antisense mRNA (AM) were listed as candidate tags. Among them, candidate sRNAs were defined as sRNAs whose expression level was $>20$ reads. The spliced tags were directly compared with 7 sRNAs with known sequences, since the database used had not incorporated the known sequences of HP. For the functional annotation of candidate sRNAs, two methods were used. Based on sequence similarity, candidate RNAs were subjected to BLAST analysis against four databases (sR NAMap (http://srnamap.mbc. nctu.edu.tw), sRNATarBase (http://ccb.bmi.ac.cn), miRBase (http://www.mirbase.org) and SIPHI (http://newbio.cs.wisc. edu). Small RNAs possessed secondary structure conservation. The secondary structures of candidate sRNAs were therefore compared using Infernal software (http://infernal.janelia.org/) and the Rfam database (http://rfam.janelia.org/) was utilized for family classification. Subsequently, the secondary structures of candidate sRNAs were analyzed by RNAfold (http:// rna.tbi.univie.ac.at/cgi-bin/RNAfold.cgi), based on minimum free energy and base-paring probability. In addition, certain concerned target genes were selected to predict candidate sRNAs (Fig. S1).

Reverse transcription-quantitative (RT-q)PCR and product sequencing. In order to detect the expression of mRNAs and sRNAs, RNA (0.2-0.5 $\mu \mathrm{g})$ was subjected to RT-PCR using the PrimeScript reverse transcription reagent kit (Takara Biotechnology Co., Ltd.) according to the manufacturer's protocol. Quantitative analysis of the expression of mRNAs and sRNAs was performed using the ABI 7300 Real-Time PCR system (Thermo Fisher Scientific, Inc.) with SYBR Premix Ex Taq ${ }^{\mathrm{TM}}$ (Takara Biotechnology Co., Ltd.). The cycling conditions were as follows: Initial incubation at $95^{\circ} \mathrm{C}$ for $15 \mathrm{sec}$, followed by 40 cycles of denaturation at $95^{\circ} \mathrm{C}$ for $5 \mathrm{sec}$ and annealing at $60^{\circ} \mathrm{C}$ for $31 \mathrm{sec} .23 \mathrm{~S}$ rRNA was used as the housekeeping gene. The primer sequences used are listed in Table I. The $2^{-\Delta \Delta \mathrm{Cq}}$ method was used to analyze the relative changes in gene expression from RT-qPCR experiments (15). The products were collected by $2 \%$ agarose gel electrophoresis, and sequencing was performed by Sangon Biotech Co., Ltd. (Shanghai, China).

Bacterial density detection. The OD600 was detected using an ultraviolet spectrophotometer (Beckman Coulter, Inc.). 
Table I. Primers used for polymerase chain reaction.

\begin{tabular}{ll}
\hline Nucleotide & \multicolumn{1}{c}{ Sequence } \\
\hline 5'-ureB-sRNA & Forward: 5'-CTTTAGCATCCATATCCGCATT-3' \\
NAT-67 & Reverse: 5'-GACTGGTGGCATTGTCACAATA-3' \\
& Forward: 5'-ACTTTTTATTGGGCGTCCTC-3' \\
ceuE & Reverse: 5'-AGAACTGGGTCCACAGCAGA-3' \\
$23 S$ rRNA & Forward: 5'-CGCAAGAAGTCAAAGTCAAGG-3' \\
& Reverse: 5'-CGCCTACAACCCTATTCCAA-3' \\
& Forward: 5'-GCTCGTGTCGTGAGATGTTG-3' \\
& Reverse: 5'-GATCGTGTAAGTGAGATGG-3'
\end{tabular}

rRNA, ribosomal RNA; sRNA, small non-coding RNA.

OD600 $=1$ was considered to indicate a corresponding density for $\mathrm{HP}$ of $10^{8} \mathrm{CFU} / \mathrm{ml}$.

Superoxide dismutase (SOD) activity. The activity of SOD in HP was determined using a Total Superoxide Dismutase Assay Kit with WST-8 (Beyotime Institute of Biotechnology). Cell lysates were prepared following various designated treatments. Assays were performed on 96-well microtiter plates by incubating $20 \mu \mathrm{l}$ cell lysate protein per sample in $160 \mu \mathrm{l}$ reaction buffer $(151 \mu \mathrm{l}$ SOD detection buffer, $8 \mu \mathrm{l}$ WST- 8 and $1 \mu$ lenzyme solution) and then adding $20 \mu \mathrm{l}$ starting work buffer $(0.5 \mu \mathrm{l}$ starting buffer and $19.5 \mu \mathrm{l} \mathrm{SOD}$ detection buffer). Lysates were incubated at $37^{\circ} \mathrm{C}$ for $30 \mathrm{~min}$. The OD450 $\mathrm{nm}$ were measured using an ultraviolet spectrophotometer (Beckman Coulter, Inc.). The procedure was performed according to the manufacturer's protocol.

ROS determination. ROS in the Hp were visualized using the dichloro-dihydro-fluorescein diacetate (DCFH-DA) fluorescent probe (Beyotime Institute of Biotechnology). In brief, HP was prepared after the respective treatment and then resuspended in DCFH-DA probe $(10 \mu \mathrm{M})$ at a density of $10^{7} \mathrm{CFU} / \mathrm{ml}$. The mixture was incubated at $37^{\circ} \mathrm{C}$ for $30 \mathrm{~min}$ and then washed with PBS buffer until clear. The ultrasonic probe was immersed into the cell suspension for ultrasonic crushing. The total ultrasonic time was $\sim 2 \mathrm{~min}$. Finally, the absorbance of cell lysates was measured at $450 \mathrm{~nm}$ (Beckman Coulter; DU800).

Statistical analysis. Data were analyzed using SPSS 17.0 (IBM, Corp.). Values are expressed as the mean \pm standard error of the mean. Analysis of variance followed by the Newman-Student-Keuls test was used to analyze the results. $\mathrm{P}<0.05$ was considered to indicate a statistically significant difference.

\section{Results}

Construction of the sRNA library of HP. The concentration of samples used for sequencing was $>150 \mathrm{ng} / \mu \mathrm{l}$, the ratio of $23 \mathrm{~S}$ rRNA to $16 \mathrm{~S}$ rRNA 1.6 was $>1.5$ and the RNA integrity number was 7.8, which met the requirements for constructing an sRNA library, according to the Small RNA Sample Preparation protocol of Illumina HiSeq2000. The 18-150 nt long fragments were purified after being excised from the gel and then sequenced by the Illumina HiSeq2000. A total of 592,125 tags were sequenced, including 84 and 234 that map to AM and the IGR, accounting for 4.3 and $11.98 \%$, respectively. Among them, 163 tags had $>20$ reads and therefore defined as candidate sRNAs and named sRNA001-sRNA163. The length distribution of the candidate sRNAs is illustrated in Fig. 1. A total of 72 candidate sRNAs were $<50 \mathrm{nt}$ long, 63 tags were 51-100 nt long, 11 tags were 101-150 nt long, 6 tags were 151-200 nt long and 11 tags were $>200 \mathrm{nt}$ long (Fig. 1A). When mapping the spliced tags to the known sequence, only 5 sRNAs [6S RNA, NAT-67, 5'ureB-sRNA, repG (HPnc5490) and NAT-39] had matching pieces; the others (IG-443 and IG-524) were undetected.

Annotation and secondary structure of candidate sRNAs in $H P$. Two methods were adopted for candidate sRNA annotation: Sequence conservation and secondary structure conservation. As presented in Fig. 1,35 sRNAs were sequence-similar and 97 were secondary structure-conservative. Among them, 30 tags were sequence-similar and secondary structure-conservative. From what has been discussed above, 61 sRNAs were reported for the first time. RNAfold (http://rna.tbi.univie.ac.at/cgi-bin/ RNAfold.cgi) was used to predict the secondary structure of candidate sRNAs. Representative results from Fig. 1B indicate that sRNA008 (112 nt) was encoded at the antisense strand, which is in opposite orientation to the IGR between HP0017 and HP0018, and sRNA099 (431 nt) was encoded at the antisense strand in the IGR between HP1143 and HP1147 (Fig. 1F). sRNA014 (66 nt) was encoded at the sense strand, opposite HP0098 (Fig. 1C). sRNA078 (76 nt) was encoded at the antisense strand, adjacent to HP0896 and HP0897 (Fig. 1G). Their secondary structures are presented in Fig. 1.

Target prediction of candidate $s R N A s$. IntaRNA was employed to forecast targets of candidate sRNAs. The strategy of this software is base-paring, leading to two further predicted targets of one sRNA, and entries with a free energy of $>-22 \mathrm{kcal} / \mathrm{mol}$ were filtered out. A total of 1,247 targets of 79 candidate sRNAs met the requirements. Therefore, sRNA interactions with genes of interest, including genes associated with oxidative stress, virulence factors and chemotaxis, were 

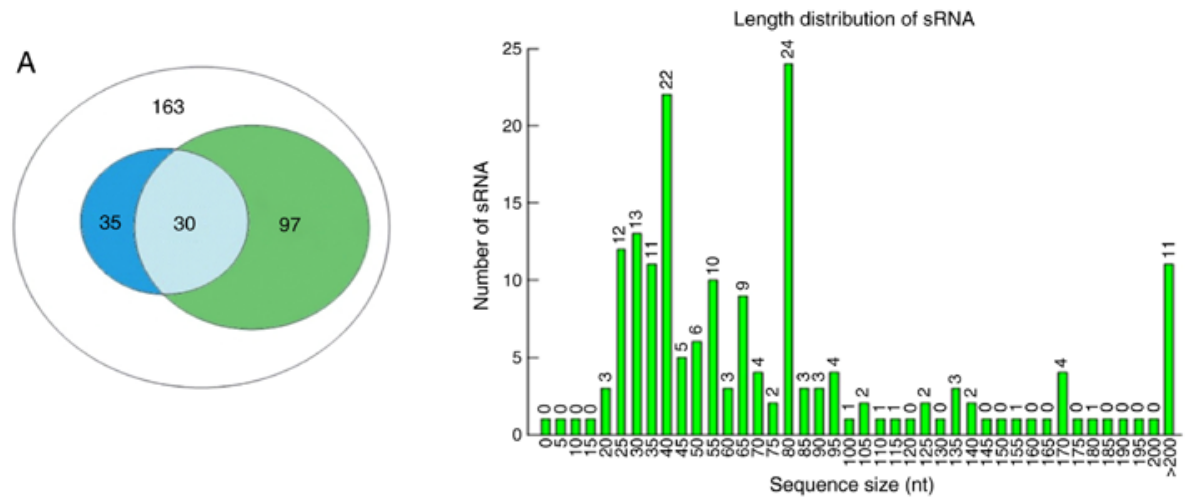

B

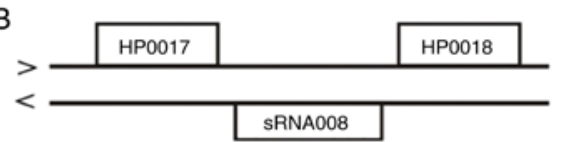

D

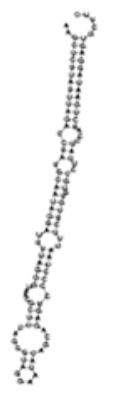

F

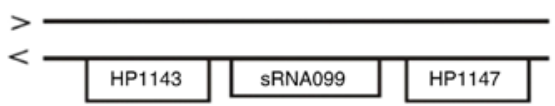

$\mathrm{H}$

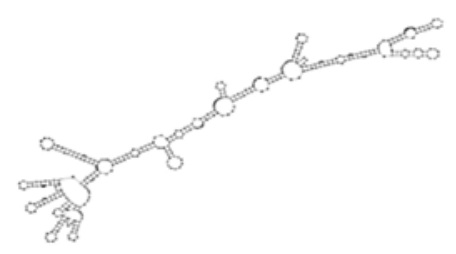

C

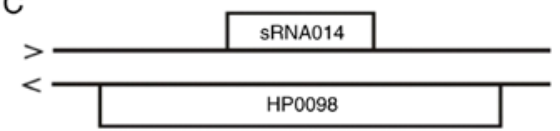

E

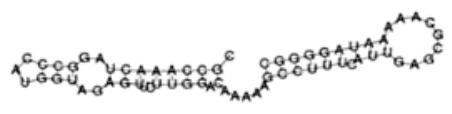

G

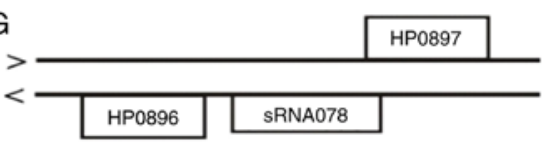

I

Figure 1. (A) Venn diagram presenting the Hp candidate sRNAs length distribution and the annotation of candidate sRNAs. Gray, total candidate sRNAs; blue, sequence conservative sRNAs; green, secondary structure evolutionarily conserved sRNAs; light blue, both conservative. Annotation and secondary structure of Hp candidate sRNAs. Gene locations of representative sRNAs were shown in (B) sRNA008, (C) sRNA014, (F) sRNA099 and (G) sRNA078. Their respective secondary structures are presented below. (D) Secondary structure of sRNA008. (E) Secondary structure of sRNA014. (H) Secondary structure of sRNA099. (I) Secondary structure of sRNA078. Hp, Helicobacter pylori; sRNA, small non-coding RNA.

predicted. The results suggested that sRNAs that may regulate virulence factors were sRNA057, sRNA126, sRNA055, sRNA121 and sRNA025 (Fig. 2A). Chemotaxis-associated genes may be regulated by sRNA057, sRNA090, sRNA055, sRNA12 and sRNA025 (Fig. 2B). The known sequence NAT-67 and sRNA057 may be associated with the regulation of oxidative stress (Fig. 2C).

Verification of NAT-67 and 5'ureB-sRNA. In order to verify the existence of HP RNAs, RT-qPCR primers for NAT-67 and 5'ureB-sRNA were designed. The DNA fragment was directly electrophoresed and sequenced. The results (Fig. 3) were consistent with previous reports $(5,8)$.
TNZ inhibits bacterial viability and downregulates NAT-67 and 5'ureB-sRNA. TNZ is used for HP treatment by inducing oxidative stress and cell death but the exact mechanism has remained to be fully elucidated. Furthermore, it has been demonstrated that certain sRNAs, including RyhB and RybA, are involved in the regulation of oxidative stress (11). Consequently, the association between TNZ-induced cell death and sRNAs was investigated. Under liquid culture conditions, the growth cycle of HP was $\sim 48 \mathrm{~h}$ (Fig. 4A). TNZ was added at the middle of the logarithmic phase $(10 \mathrm{~h})$, since, during this phase, sRNAs begin to be expressed and the response of bacteria to environmental stimuli is at its most sensitive. The growth of HP was significantly suppressed following 


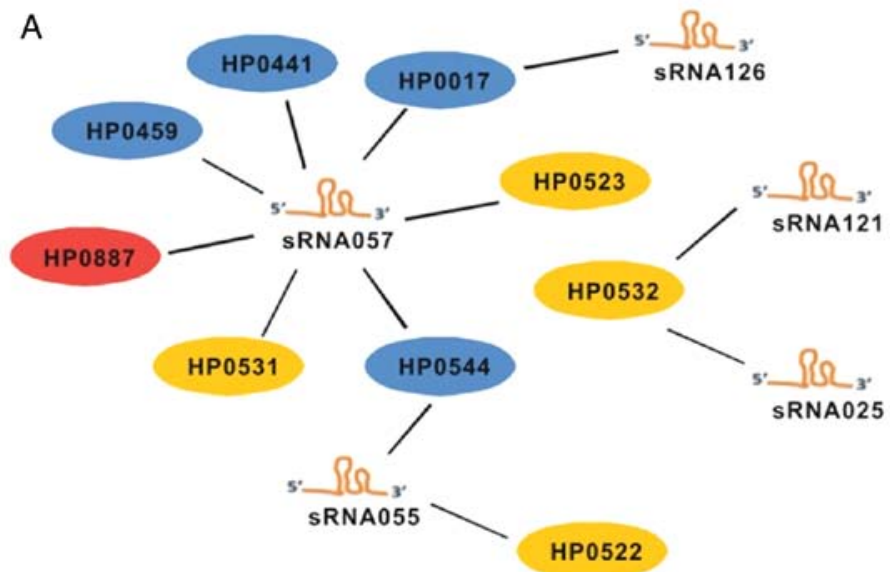

B

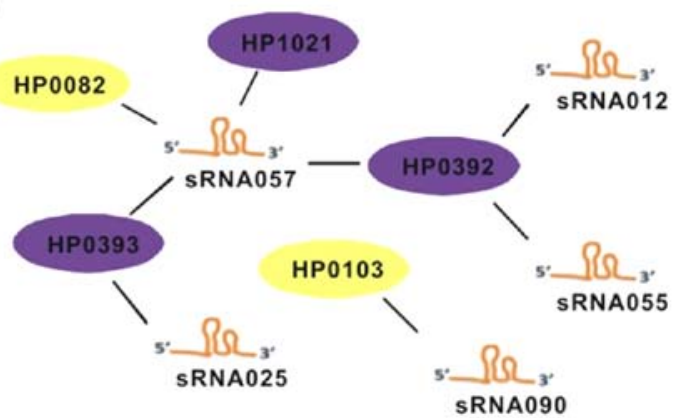

C

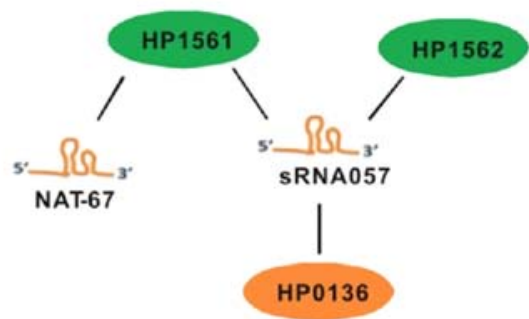

Figure 2. Target prediction of candidate sRNAs. Targets associated with (A) virulence factors, (B) chemotaxis and (C) oxidative stress are provided. Blue, type IV secretion system-associated genes; yellow, cag-pathogenicity island protein; red, vacuolating cytotoxin-associated genes; purple, chemotaxis protein-encoding genes; pale yellow, methyl-accepting chemotaxis protein-associated genes; green, ceuE; orange, peroxidase. Hp, Helicobacter pylori; sRNA, small non-coding RNA.
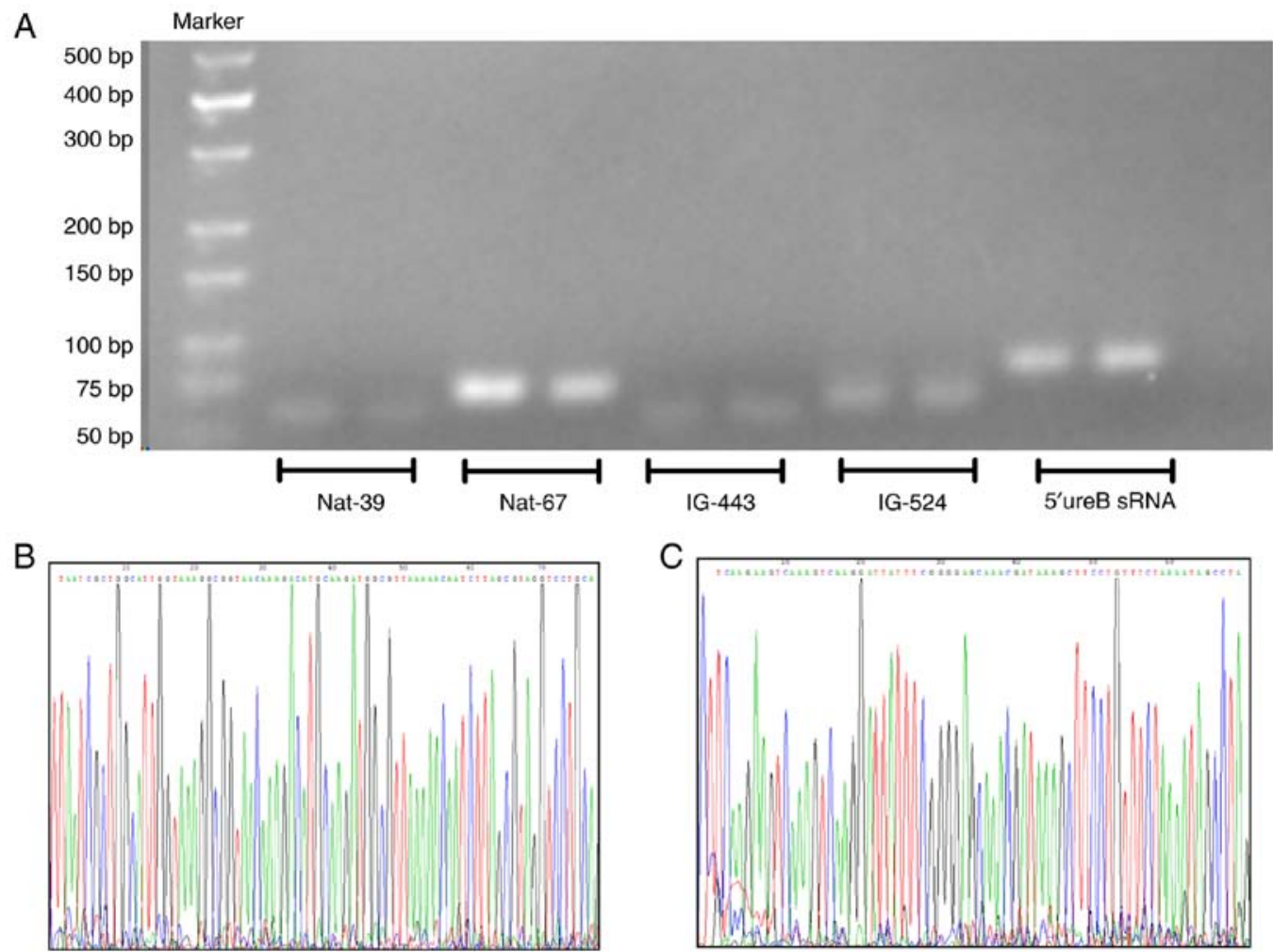

Figure 3. Verification of five known sRNA of Hp including Nat-39, Nat-67, IG-443, IG-524 and 5'ureB-sRNA. (A) Reverse transcription-quantitative PCR was performed, and the amplified products of five sRNAs were electrophoresed in a $2 \%$ agarose gel. 5'ureB-sRNA and Nat-67 can be detected clearly and Nat-39, IG-443 and IG-524 were weakly detected. (B) 5'ureB-sRNA PCR products were sequenced. (C) Nat-67 PCR products were sequenced. sRNA, small non-coding RNA; Hp, Helicobacter pylori. 

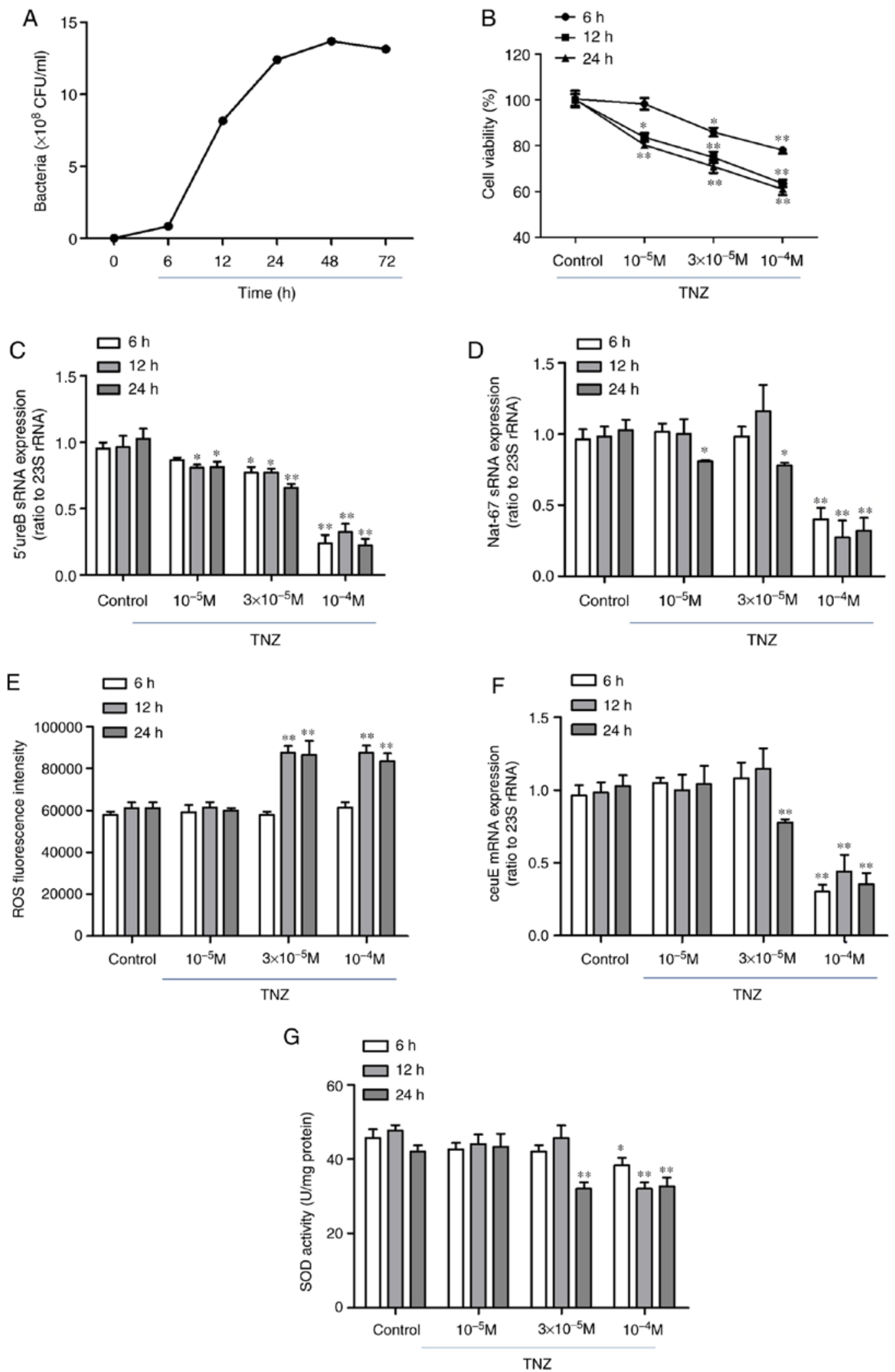

Figure 4. Effect of TNZ on the expression of sRNA and oxidative stress levels in HP. (A) Growth curve of Hp SS1. (B) Cell viability in the presence of TNZ was determined with an ultraviolet spectrophotometer. (C and D) The expression of (C) 5'ureB-sRNA and (D) NAT-67 was determined by RT-qPCR. (E) ROS levels. (F) The expression of ceuE was detected by RT-qPCR. (G) SOD activity. Values are expressed as the mean \pm standard error of the mean (n=5), ${ }^{*} \mathrm{P}<0.05,{ }^{* *} \mathrm{P}<0.01$ vs. Control. CFU, colony-forming units; RT-qPCR, reverse transcription-quantitative PCR; ROS, reactive oxygen species; SOD, superoxide dismutase; Hp, Helicobacter pylori; sRNA, small non-coding RNA; TNZ, tinidazole.

treatment with TNZ, particularly at $12 \mathrm{~h}$ (Fig. 4B). Of note, the expression of NAT-67 and 5'ureB-sRNA was significantly reduced (Fig. 4C and D).
TNZ downregulates ceuE and induces oxidative stress. It was reported that NAT-67 may be the regulator of iron-binding protein ceuE, which may participate in the regulation of 
oxidative stress. The expression of ceuE and oxidative stress in TNZ-treated Hp were therefore observed. It was indicated that TNZ significantly decreased ceuE expression and SOD activity, while increasing ROS levels (Fig. 4E-G). These results suggested that the induction of cell death by TNZ may be achieved through the downregulation of NAT-67 and induction of oxidative stress.

\section{Discussion}

The significance of sRNA (50-500 nt in length) in the regulation of bacteria is becoming increasingly apparent. These molecules are involved in the regulation of a wide range of physiological responses, cellular metabolism and reactions to environmental stimuli (e.g., $\mathrm{pH}$ or temperature shifts), ultimately contributing to survival and virulence (16). It has been demonstrated that sRNA is involved in the response of bacteria to environmental fluctuations, including oxidative stress and antibiotic drug exposure. For instance, azithromycin inhibits the expression of small RNAs RsmY and RsmZ in $P$. aeruginosa, which may be a bactericidal mechanism (14).

Hp, which specifically colonizes the gastric epithelium, is the dominant pathogenic factor for chronic gastric ulcer and gastric cancer. Recent studies have indicated that sRNAs were crucial to the growth, colonization and pathogenicity of Hp. For instance, nucleotide pairing revealed that Hp sRNA NAT-67 was completely complementary to periplasmic iron-binding protein HP1561 (ceuE), suggesting that NAT-67 may be a regulator in iron homeostasis and oxidative stress (8). 5'ureB-sRNA, a cis-encoded antisense small RNA regulated by the HP0165-HP0166 two-component system in Hp, enhanced expression of ureB, consequently protecting it from acid $(7,10)$. Furthermore, 5'ureB knockout in Hp markedly inhibited the inflammatory response of gastric mucosa epithelial cells (17). However, at present, the full extent of sRNAs in $\mathrm{Hp}$ chromosomes remains elusive. While bioinformatics prediction indicated that hundreds of sRNAs may exist in $\mathrm{Hp}$, only 7 sRNAs had a known nucleotide sequence $(5,8-9)$. In the present study, the sRNA expression spectrum was reported for the first time. The results provided 163 candidate sRNAs in Hp. Among the 7 known sRNAs, 5, including 6S RNA, NAT-67, 5'ureB-sRNA, repG (HPnc5490) and NAT-39, matched the sequence in the present study, while IG-443 and IG-524 were undetected. A possible reason for this is that sRNA varies with time and environment. Furthermore, RNAfold was used for secondary structure and IntaRNA for target prediction. Based on base-paring, the so-called predicted targets with a free energy of below $-22 \mathrm{kcal} / \mathrm{mol}$ were filtered out. In order to obtain a more specific result, the interactions between sRNAs and genes of interest, including genes associated with oxidative stress, virulence factors and chemotaxis, were predicted. It was indicated that several sRNAs, including NAT-67, are highly correlated with these genes. Bioinformatics analysis of $\mathrm{HP}$ is momentous and may facilitate future research on sRNA and understanding the pathogenic mechanism of HP.

In the present study, bioinformatics analysis and gene sequencing were combined to determine gene location, sequence similarity and secondary structure. However, this approach had its limitations in predicting series of sRNAs.
First, the sRNAs screened in the present study were located in IGRs and AM, but some were encoded by open reading frames and untranslated regions (UTRs), and therefore, certain tags may have been missed. In addition, it is required to analyze the initiator and terminator, as the candidate sRNAs were short clips. However, the major limitation is the reliability and repeatability of the bioinformatics analysis, the results of which require to be confirmed. In the present study, RT-qPCR and sequencing were performed. The northern blot and gene chip would be the choices in further studies.

NAT-67 is involved in regulating ceuE (HP1561) and ceuE is associated with HP growth (9). For this reason, the influence of TNZ, a frequently-used clinical drug for HP infection, on sRNA expression and bacterial growth was assessed. TNZ suppressed Hp growth while downregulating NAT-67 and ceuE. However, the effects of sRNA regulation depend on the binding site of target mRNAs. If the binding site contains a ribosome binding site, it prevents $30 \mathrm{~S}$ ribosomes from binding to mRNA, which suppresses transcription or degrades mRNA (18). On the other hand, if the binding site of mRNA forms a specific secondary structure, combination with sRNA results in hairpin opening, so that mRNA stabilizes and/or promotes translation (4). E. coli sRNA RyhB binding to the 5'-UTR of cirA increased mRNA stability and translation (19). $S$. aureus sRNA FasX combining with a virulence-associated gene also enhanced its activity (20). These results supported that TNZ exerts its antibacterial effect by downregulating NAT-67 and then inhibiting ceuE.

HP1561 (ceuE), a periplasmic iron-binding protein that binds and transports iron into cell, has an important role in iron homeostasis. NAT-67 (90 nt) was encoded in the IGR between gene ftsW and tsaA, with perfect complementarity to HP1561 (ceuE). Iron is the key ion for survival and growth. Hp expresses only a single SOD, the iron-cofactor one, known as SODB (21). Therefore, a decrease in the cellular iron concentration of HP leads to a decrease in SOD activity and increase in oxidative stress. The present study indicated that TNZ exposure restrained NAT-67 and ceuE expression, accompanied by a SOD activity decrease and ROS increase. These results provided novel experimental evidence with regards to the antimicrobial mechanism of TNZ.

It is now accepted that miRNAs have crucial regulatory roles in almost all pathological processes by regulating cell growth, proliferation, differentiation and apoptosis. Recent results have suggested that miRNAs not only execute functions within the original cells but may also be initiatively secreted to regulate adjacent or distant cells functions, and are even transmitted from one species to another, facilitating crosstalk, communication or signal interference between them (22-24). For example, Rhodovulum sulfidophilum bacterial extracellular RNAs have been reported in the literature (25). Nucleic acids were detected in Lactobacillus culture filtrate and the major group were RNAs (including sRNAs) (26). Studies have reported that sequence-similar miRNAs may coordinately regulate one target mRNA and thus, microRNA-mediated miRNA regulation may be a novel model (27). For instance, cardiac miR-378 and miR-499 regulated miR-143 (28-29). It may therefore be speculated that bacteria-secreted sRNAs may be a novel pathogenic factor. 


\section{Acknowledgements}

Not applicable.

\section{Funding}

This work was supported by the National Natural Science Foundation of China (grant nos. 81703592 to JD and 81573486 to YJL). It was also supported by the Natural Science Foundation of Hunan province (grant no. 2019JJ50934) and the Open Sharing Fund for the Large-scale Instruments and Equipments of Central South University.

\section{Availability of data and materials}

The datasets used and/or analyzed during the current study are available from the corresponding author on reasonable request.

\section{Authors' contributions}

JD, XL and YL designed the present study; JD and WZ performed the experiments; JD and WZ analyzed the data and wrote this manuscript. JD and WZ contributed to the manuscript equally. All authors read and approved the final manuscript.

\section{Ethics approval and consent to participate}

Not applicable.

\section{Patient consent for publication}

Not applicable.

\section{Competing interests}

The authors declare that they have no competing interests.

\section{References}

1. Salama NR, Hartung ML and Müller A: Life in the human stomach: Persistence strategies of the bacterial pathogen Helicobacter pylori. Nat Rev Microbiol 11: 385-399, 2013.

2. Posselt G, Backert $\mathrm{S}$ and Wessler S: The functional interplay of Helicobacter pylori factors with gastric epithelial cells induces a multi-step process in pathogenesis. Cell Commun Signal 11: 77, 2013.

3. Wassarman KM: 6S RNA: A small RNA regulator of transcription. Curr Opin Microbiol 10: 164-168, 2007.

4. Lalaouna D, Simoneau-Roy M, Lafontaine D and Massé E: Regulatory RNAs and target mRNA decay in prokaryotes. Biochim Biophys Acta 1829: 742-747, 2013.

5. Sharma CM, Hoffmann S, Darfeuille F, Reignier J, Findeiss S, Sittka A, Chabas S, Reiche K, Hackermüller J, Reinhardt R, et al The primary transcriptome of the major human pathogen Helicobacter pylori. Nature 464: 250-255, 2010.

6. Pernitzsch SR, Tirier SM, Beier D and Sharma CM: A variable homopolymeric G-repeat defines small RNA-mediated posttranscriptional regulation of a chemotaxis receptor in Helicobacter pylori. Proc Natl Acad Sci USA 111: E501-E510, 2014.

7. Wen Y, Feng J and Sachs G: Helicobacter pylori 5'ureB-sRNA, a cis-encoded antisense small RNA, negatively regulates ure $\mathrm{AB}$ expression by transcription termination. J Bacteriol 195: 444-452, 2013

8. Xiao B, Li W, Guo G, Li BS, Liu Z, Tang B, Mao XH and Zou QM: Screening and identification of natural antisense transcripts in Helicobacter pylori by a novel approach based on RNase I protection assay. Mol Biol Rep 36: 1853-1858, 2009.
9. Xiao B, Li W, Guo G, Li B, Liu Z, Jia K, Guo Y, Mao X and Zou Q: Identification of small noncoding RNAs in Helicobacter pylori by a bioinformatics based approach. Curr Microbiol 58: 258-263, 2009.

10. Yi W, Jing F, Scott DR, Marcus EA and Sachs G: A cis-encoded antisense small RNA regulated by the HP0165-HP0166 two-component system controls expression of ureB in Helicobacter pylori. J bacterial 193: 40-51, 2011.

11. Calderón IL, Morales EH, Collao B, Calderón PF, Chahuán CA, Acuña LG, Gil F and Saavedra CP: Role of salmonella typhimurium small RNAs RyhB-1 and RyhB-2 in the oxidative stress response. Res Microbiol 165: 30-40, 2014.

12. Howden BP, Beaume M, Harrison PF, Hernandez D, Schrenzel J, Seemann T, Francois P and Stinear TP: Analysis of the small RNA transcriptional response in multidrug-resistant staphylococcus aureus after antimicrobial exposure. Antimicrob Agents Chemother 57: 3864-3874, 2013.

13. Eyraud A, Tattevin P, Chabelskaya S and Felden B: A small RNA controls a protein regulator involved in antibiotic resistance in staphylococcus aureus. Nucleic Acids Res 42: 4892-4905, 2014.

14. Pérez-Martínez I and Haas D: Azithromycin inhibits expression of the GacA-dependent small RNAs RsmY and RsmZ in pseudomonas aeruginosa. Antimicrob Agents Chemother 55: 3399-3405, 2011.

15. Livak KJ and Schmittgen TD: Analysis of relative gene expression data using real-time quantitative PCR and the 2(-Delta Delta $\mathrm{C}(\mathrm{T})$ ) method. Methods 25: 402-408, 2001.

16. Wassarman KM: Small RNAs in bacteria: Diverse regulators of gene expression in response to environmental changes. Cell 109: 141-144, 2002.

17. Perrais M, Rousseaux C, Ducourouble MP, Courcol R, Vincent $P$, Jonckheere $\mathrm{N}$ and Van Seuningen I: Helicobacter pylori urease and flagellin alter mucin gene expression in human gastric cancer cells. Gastric Cancer 17: 235-246, 2014.

18. Desnoyers G, Bouchard MP and Massé E: New insights into small RNA-dependent translational regulation in prokaryotes. Trends Genet 29: 92-98, 2013.

19. Salvail H, Caron MP, Bélanger J and Massé E: Antagonistic functions between the RNA chaperone $\mathrm{Hfq}$ and an sRNA regulate sensitivity to the antibiotic colicin. EMBO J 32: 2764-2778, 2013.

20. Ramirez-Peña E, Treviño J, Liu Z, Perez N and Sumby P: The group A Streptococcus small regulatory RNA FasX enhances streptokinase activity by increasing the stability of the ska mRNA transcript. Mol Microbiol 78: 1332-1347, 2010.

21. Tsugawa H, Suzuki H, Satoh K, Hirata K, Matsuzaki J, Saito Y, Suematsu M and Hibi T: Two amino acids mutation of ferric uptake regulator determines Helicobacter pylori resistance to metronidazole. Antioxid Redox Signal 14: 15-23, 2011.

22. Liang H, Zen K, Zhang J, Zhang CY and Chen X: New roles for microRNAs in cross-species communication. RNA Biol 10: 367-370, 2013.

23. Scott RB, Christopher N, Xie F, Wood JR and Zempleni J: MicroRNAs are absorbed in biologically meaningful amounts from nutritionally relevant doses of cow milk and affect gene expression in peripheral blood mononuclear cells, HEK-293 kidney cell cultures, and mouse livers. J Nutr 144: 1495-1500, 2013.

24. Zhou Z, Li X, Liu J, Dong L, Chen Q, Liu J, Kong H, Zhang Q, Qi X,Hou D, et al: Honeysuckle-encoded atypical microRNA2911 directly targets influenza A viruses. Cell Res 25: 39-49, 2015.

25. Li J, Zhang Y, Li D, Liu Y, Chu D, Jiang X, Hou D, Zen K and Zhang CY: Small non-coding RNAs transfer through mammalian placenta and directly regulate fetal gene expression. Protein Cell 6: 391-396, 2015.

26. Ando T, Suzuki H, Nishimura S, Tanaka T, Hiraishi A and Kikuchi Y: Characterization of extracellular RNAs produced by the marine photosynthetic bacterium rhodovulumsulfidophilum. J Biochem 139: 805-811, 2006.

27. Li T, Ling ZX and Wen S: Analysis of nucleic acids in lactobacillus culture filtrate. Chin J Microecol 23: 104-106, 2011.

28. Guo L, Sun B, Wu Q, Yang S and Chen F: miRNA-miRNA interaction implicates for potential mutual regulatory pattern. Gene 511: 187-194, 2012.

29. Matkovich SJ, Hu Y and Dorn GW II: Regulation of cardiac microRNAs by cardiac microRNAs. Circ Res 113: 62-71, 2013.

This work is licensed under a Creative Commons Attribution-NonCommercial-NoDerivatives 4.0 International (CC BY-NC-ND 4.0) License. 